\title{
Should surgical ablation for atrial fibrillation be performed in patients with a significantly enlarged left atrium?
}

\author{
Niv Ad, MD, Linda Henry, PhD, Sharon Hunt, MBA, and Sari D. Holmes, PhD
}

\begin{abstract}
Objective: One established predictor for failure of surgical ablation for atrial fibrillation is increased left atrial size. Surgeon perception is that surgical ablation in these patients is ineffective and should not be performed. The purpose of this study was to determine whether a larger left atrial size carries a prohibitive risk for failure and embolic events after surgical ablation.
\end{abstract}

\begin{abstract}
Methods: In patients undergoing surgical ablation without left atrial reduction $(\mathrm{N}=373)$, left atrial size was measured via transthoracic echocardiography within 6 months before surgery. Large $(>5.5 \mathrm{~cm} ; \mathrm{n}=83)$ and small $(\leq 5.5 \mathrm{~cm} ; \mathrm{n}=290)$ left atrial size groups were compared on outcomes.

Results: Patients in the large left atrium group were younger $(P=.02)$ and had lower operative risk (European System for Cardiac Operative Risk Evaluation, $P=.01$ ), but they were not different in type $(P=.51)$ or duration of atrial fibrillation $(P=.93)$. The large left atrium group was less likely to be in sinus rhythm at 1 year $(86 \%$ vs $93 \%, P=.04)$, but there was no difference in sinus rhythm without antiarrhythmic drugs $(77 \%$ vs $85 \%, P=.10)$. By 2 years, the large and small left atrium groups were similar in sinus rhythm $(85 \%$ vs $90 \%, P=.35)$. Freedom from embolic stroke was $\operatorname{similar}(P=.70)$ despite the majority of patients not taking anticoagulation at 1 year.

Conclusions: The large left atrium group had acceptable return to sinus rhythm and sinus rhythm without antiarrhythmic drugs. The embolic stroke rate was low despite the majority of patients not taking anticoagulation. If patients are managed appropriately post-ablation, left atrial size should not be a discouragement when evaluating surgical candidates with atrial fibrillation. (J Thorac Cardiovasc Surg 2014;147:236-41)
\end{abstract}

The first Cox-Maze procedure for surgical ablation of atrial fibrillation (AF) was performed more than 25 years ago, and despite some changes in the lesion set, the basic concepts of the procedure have remained the same. However, the advent of new technology has provided an opportunity for the lesions to be completed with alternate energy sources, primarily cryothermia and radiofrequency, allowing for less time spent on bypass and a less tedious procedure. ${ }^{1-5}$

Current long-term results for surgical ablation of $\mathrm{AF}$ range from $70 \%$ to $90 \%$ depending on whether any modifications to the original lesion set were made, especially in patients with nonparoxysmal $\mathrm{AF}^{2,6}$ Along with the reporting of results, many investigators have reported the predictors of failure in their cohort of patients. ${ }^{7-11}$ A consistent finding for most investigators has been the size of the left atrium (LA) at the time of surgery, such that the larger the LA $(>5.5 \mathrm{~cm})$ the higher the rate

From the Inova Heart and Vascular Institute, Falls Church, Va.

Disclosures: Niv Ad reports consulting fees from Estech, Medtronic, and Atricure. The other authors have nothing to disclose with regard to commercial support.

Read at the 39th Annual Meeting of The Western Thoracic Surgical Association, Coeur d'Alene, Idaho, June 26-29, 2013.

Received for publication June 26, 2013; revisions received Sept 6, 2013; accepted for publication Sept 17, 2013; available ahead of print Nov 4, 2013.

Address for reprints: Niv Ad, MD, Cardiac Surgery, Inova Heart and Vascular Institute, Falls Church, VA 22042 (E-mail: Niv.Ad@inova.org).

$0022-5223 / \$ 36.00$

Copyright (c) 2014 by The American Association for Thoracic Surgery

http://dx.doi.org/10.1016/j.jtcvs.2013.09.037 of failure. ${ }^{7-11}$ As a result, the perception among surgeons is that surgical ablation in patients with an enlarged LA is highly ineffective and should not be performed. There are no clear data on whether certain LA dimensions are related to results and thereby prohibit surgical ablation.

The purpose of this study was to explore the variables associated with failure of the full Cox-Maze procedure as originally designed. ${ }^{1,12}$ Specifically, we sought to (1) examine the traditional predictors of failure (age, gender, left atrial size, and duration of $\mathrm{AF}$ ) at 12 months to determine the relevancy when newer ablative devices are used; and (2) determine whether preoperative left atrial size greater than $5.5 \mathrm{~cm}$ carries a risk for recurrent atrial arrhythmia and embolic events after surgical ablation.

\section{MATERIALS AND METHODS}

This was a cohort study whereby all patients with preoperative left atrial size measurement who underwent surgical ablation without left atrial reduction surgery between January 2005 and August 2012 were followed prospectively $(\mathrm{N}=518)$. A total of 373 consecutive patients were available for analysis with follow-up data at 1 year. There were 145 patients who did not complete 1-year follow-up, in whom there were 6 operative deaths ( $<30$ days; $1.2 \%)$ and 21 deaths during the first year of follow-up.

All patients were included in our unique AF registry, which stores detailed information collected prospectively about the surgical ablation case including preoperative and postoperative data. Rhythm status using electrocardiography and 24-hour Holter, and clinical follow-up were collected and verified at 3, 6, 9, 12,18, and 24 months and yearly thereafter. The definition for success or failure of surgical ablation was according to 


\section{Abbreviations and Acronyms \\ $\mathrm{AF}=$ atrial fibrillation \\ $\mathrm{CI}=$ confidence interval \\ $\mathrm{LA}=$ left atrium \\ $\mathrm{OR}=$ odds ratio \\ $\mathrm{SR}=$ sinus rhythm}

the Heart Rhythm Society guidelines. ${ }^{13}$ Data from this AF registry were merged with data from the Society of Thoracic Surgeons database at the Inova Heart and Vascular Institute. Left atrial size was measured via transthoracic echocardiography within 6 months before surgery. All patients underwent standard transthoracic examination using the routine protocols from our accredited cardiac diagnostics department. The anteroposterior left atrial diameter was measured from the parasternal long-axis view using M-mode measurements. All perioperative outcomes were determined according to the Society of Thoracic Surgeons database definitions. This study was approved by the institutional review board at the Inova Heart and Vascular Institute, and a waiver of patient consent for our research program was obtained (studies 06.022 and 12.055).

\section{Surgical Ablation Technique}

Surgical ablation was performed by multiple surgeons. In $87 \%$ of patients, the complete Cox-Maze III/IV lesion set was performed as described previously. ${ }^{7,12}$ In the remaining $13 \%$ of patients, pulmonary vein isolation or limited left-sided surgical ablation was performed in patients presenting with intermittent AF (paroxysmal or persistent $\mathrm{AF}$ ). Cryothermia only (Medtronic, Inc, Minneapolis, Minn) was used as the sole energy source in $44 \%$ of patients, bipolar radiofrequency was used in $10 \%$ of patients, and a combination of cryothermia and bipolar radiofrequency (Atricure, West Chester, Ohio) was used in the remaining $46 \%$ of patients.

\section{Statistical Analysis}

Continuous data are presented as mean \pm standard deviation, and categorical data are presented as frequency (percent) unless otherwise noted. All analyses were conducted using SPSS version 17.0 (SPSS Inc, Chicago, Ill). Group comparisons for perioperative and rhythm outcomes were conducted in 2 groups based on left atrial size: large $(>5.5 \mathrm{~cm})$ and small $(\leq 5.5 \mathrm{~cm})$. There are multiple publications related to left atrial size as a predictor for failure in electrophysiology and surgical literature. The left atrial sizes mentioned are usually between 5 and $6 \mathrm{~cm} .{ }^{14}$ Therefore, we chose $5.5 \mathrm{~cm}$ as the reference cutoff.

Categorical variables were compared using the chi-square test or Fisher's exact test, and continuous measures were examined with Student's independent samples $t$ test or Mann-Whitney $U$ test as appropriate, based on parametric test assumptions. The impact of left atrial size (as a continuous measure) on rhythm status also was analyzed using univariate logistic regression. Failure in these analyses was defined as recurrence of $\mathrm{AF}$ at the time point of interest. In addition, multivariate logistic regression was conducted to examine the predictors of failure at 1 year in all patients and within the large LA group only. The factors included in this model were age, gender, diabetes, ejection fraction (\%), peripheral vascular disease, hypertension, additive European System for Cardiac Operative Risk Evaluation, duration of AF (years), ablation energy source, surgical ablation lesion set (full vs limited), number of concomitant surgeries, and left atrial size as a continuous variable (for the full sample analysis only).

\section{RESULTS}

\section{Patient Characteristics}

Since 2005, there were 373 patients with surgical ablation and 1-year follow-up. The large LA group $(>5.5 \mathrm{~cm})$ included 83 patients, and the small LA group $(\leq 5.5 \mathrm{~cm})$ included 290 patients. The large LA group was younger $(P=.02)$, had lower operative risk (European System for Cardiac Operative Risk Evaluation, $P=.01$ ), had fewer patients with diabetes mellitus $(P=.02)$ and hypertension $(P=.02)$, had lower ejection fraction $(P=.03)$, and had significantly more concomitant mitral valve surgery $(P<.001 ;$ Table 1$)$. There were no differences in type or duration of AF between the groups $(P=.93)$.

\section{Perioperative Morbidity}

Between January 2005 and August 2012, a total of 518 patients with preoperative transthoracic echocardiography and left atrial size measurements underwent a surgical ablation procedure. A total of 145 patients were excluded from the analysis, in whom there were 6 operative deaths $(<30$ days; $1.2 \%$ ) and 21 deaths during the first year of followup, leaving 373 patients for the final analysis. Perioperatively, the large LA group was not different from the small LA group on permanent stroke ( $0 \%$ vs $0.7 \%, P=1.00)$, prolonged ventilation greater than 24 hours $(5 \%$ vs $6 \%$, $P=.80)$, pneumonia $(1 \%$ vs $3 \%, P=.69)$, reoperation for bleeding $(1 \%$ vs $5 \%, P=.32)$, new renal failure $(4 \%$ vs $2 \%, P=.38)$, new renal failure requiring dialysis $(2 \%$ vs $1 \%, P=.31)$, and readmission within 30 days $(10 \%$ vs $12 \%, 0.70)$.

\section{Sinus Rhythm Outcomes}

Results indicated that the large LA group was less likely to be in sinus rhythm (SR) at 1 year compared with the small LA group ( $86 \%$ vs $93 \%, P=.04$; Table 2 ). However, the large LA group was not different from the small LA group in terms of SR without class I/III antiarrhythmic drugs at 1 year $(77 \%$ vs $85 \%, P=.10)$. By 2 years, the large LA group was similar to the small LA group in SR overall $(85 \%$ vs $90 \%, P=.35)$ and SR without antiarrhythmic drugs $(73 \%$ vs $81 \%, P=.28)$. Furthermore, at 1 year, patients with left atrial size $7.5 \mathrm{~cm}$ or greater $(\mathrm{n}=7)$ showed reasonable rates of SR $(86 \%)$ and SR without antiarrhythmic drugs $(71 \%)$. The large LA group did not require more cardioversions ( $23 \%$ vs $18 \%, P=.34$ ) during follow-up.

The incidence of embolic stroke was low in both the small $(\mathrm{n}=5,1.7 \%)$ and large $(\mathrm{n}=1,1.2 \%)$ LA groups. Freedom from embolic stroke during follow-up was found to be similar across LA groups $(\log$ rank $=0.15, P=.70$; Figure 1) despite the majority of patients in both the small and large LA groups not taking warfarin at 12 months $(67 \%$ vs $61 \%, P=.35)$. The majority of patients who remained on warfarin at 12 months were treated with anticoagulation for non-AF indications (ie, deep vein thrombosis, pulmonary embolism), and this did not differ significantly across LA groups $(55 \%$ vs $71 \%, P=.14$; Table 2). 
TABLE 1. Patient characteristics

\begin{tabular}{lccc}
\hline & $\begin{array}{c}\text { Small LA } \\
(\mathbf{n}=\mathbf{2 9 0})\end{array}$ & $\begin{array}{c}\text { Large LA } \\
(\mathbf{n}=\mathbf{8 3})\end{array}$ & $\begin{array}{c}\boldsymbol{P} \\
\text { value }\end{array}$ \\
\hline Age, y & $65.3 \pm 11.0$ & $61.9 \pm 11.8$ & .02 \\
Male & $201(69)$ & $52(63)$ & .25 \\
EF (\%) & $55.9 \pm 10.8$ & $52.4 \pm 12.7$ & .03 \\
CHF & $93(32)$ & $31(37)$ & .37 \\
Diabetes & $52(18)$ & $6(7)$ & .02 \\
CPD & $35(12)$ & $10(12)$ & 1.00 \\
Hypertension & $194(67)$ & $44(53)$ & .02 \\
PVD & $19(7)$ & $5(6)$ & 1.00 \\
Additive EuroSCORE & $5.7 \pm 2.7$ & $5.0 \pm 2.3$ & .01 \\
Duration of AF (mo) & $41.3 \pm 56.0$ & $41.9 \pm 50.6$ & .93 \\
Type of AF & & & \\
$\quad$ Long-standing persistent & $154(53)$ & $48(58)$ & .45 \\
Persistent & $110(38)$ & $25(30)$ & .19 \\
Paroxysmal/other & $26(9)$ & $10(12)$ & .40 \\
Full Cox-Maze & $245(85)$ & $79(95)$ & .009 \\
Minimally invasive & $228(79)$ & $60(72)$ & .23 \\
Cryothermia energy source & $129(45)$ & $35(42)$ & .71 \\
Concomitant CABG & $87(30)$ & $16(19)$ & .054 \\
Concomitant aortic valve & $70(24)$ & $11(13)$ & .04 \\
Concomitant mitral valve & $111(38)$ & $53(64)$ & $<.001$ \\
\hline AF, A & &
\end{tabular}

$A F$, Atrial fibrillation; $C A B G$, coronary artery bypass grafting; $C H F$, congestive heart faillure; $C P D$, chronic pulmonary disease; $E F$, ejection fraction; EuroSCORE, European System for Cardiac Operative Risk Evaluation; $L A$, left atrium; $P V D$, peripheral vascular disease.

\section{Predictors of Failure}

Univariate analysis using left atrial size as a continuous variable (range, 2.2-11.0 cm; Figure 2) found that the predicted probability of failure at 1 year was $43 \%$ greater with each $1-\mathrm{cm}$ increase in left atrial size (odds ratio [OR], $1.43 ; 95 \%$ confidence interval $[\mathrm{CI}], 1.06-1.92 ; P=.02$; Figure 3). Predicted probability of failure at 1 year without antiarrhythmic drugs was $30 \%$ greater with each $1-\mathrm{cm}$ increase in left atrial size (OR, 1.30; 95\% CI, 1.02-1.65; $P=.03$ ). Figure 4 displays the change in slope for the univariate relationship between left atrial size and failure as left atrial size increases. Of note, the slope between left atrial size of $7.5 \mathrm{~cm}$ and $8 \mathrm{~cm}$ changes considerably for both failure and failure without antiarrhythmic drugs at 1 year. In addition, the slope of the relationship with failure at 1 year appears to shift upward between 5 and $6 \mathrm{~cm}$. This point

TABLE 2. Sinus rhythm outcomes

\begin{tabular}{lrrr}
\hline & $\begin{array}{c}\text { Small LA } \\
(\mathbf{n = 2 9 0 )}\end{array}$ & $\begin{array}{c}\text { Large LA } \\
(\mathbf{n}=\mathbf{8 3})\end{array}$ & $\begin{array}{c}\boldsymbol{P} \\
\text { value }\end{array}$ \\
\hline NSR - 1 y & $270(93)$ & $71(86)$ & .04 \\
NSR off AAD - 1 y & $247(85)$ & $64(77)$ & .10 \\
NSR - 2 y & $174 / 193(90)$ & $51 / 60(85)$ & .35 \\
NSR off AAD - 2 y & $155 / 192(81)$ & $44 / 60(73)$ & .28 \\
Cardioversion during follow-up & $52(18)$ & $19(23)$ & .34 \\
Warfarin - 1 y & $94 / 284(33)$ & $31 / 79(39)$ & .35 \\
$\quad$ Non-AF clinical indication & $52 / 94(55)$ & $22 / 31(71)$ & .14 \\
\hline$A A D$, Antiarrhythmic drug; $A F$, atrial fibrillation; $L A$, left atrium; $N S R$, normal sinus \\
rhythm.
\end{tabular}

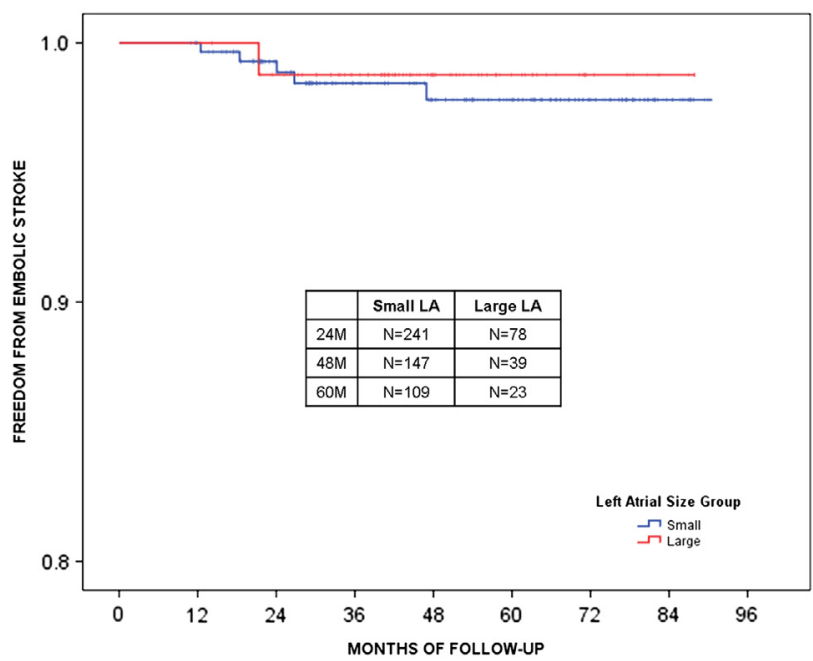

FIGURE 1. Comparison of freedom from embolic stroke indicating no difference between the LA groups ( $\log$ rank $=0.15, P=.70$ ). $L A$, Left atrium.

indicates that LA groups based on a cut-point of $5.5 \mathrm{~cm}$ presents a reasonable group differentiation.

Multivariate analysis of all traditional predictors of rhythm status (including left atrial size as a continuous variable) indicated that the only significant predictors of failure at 1 year were duration of $\mathrm{AF}$ in years (OR, 1.13; $95 \% \mathrm{CI}, 1.05-1.22 ; P<.001)$ and number of concomitant surgical procedures (OR, 1.74; 95\% CI, 1.04-2.90; $P=.03$ ), but not left atrial size (OR, $1.28 ; 95 \% \mathrm{CI}, 0.88-$ $1.87 ; P=.20)$, type of ablation procedure (OR, 1.87 ; $95 \% \mathrm{CI}, 0.36-9.72 ; P=.46$ ), or energy source (OR, 0.66 ; $95 \% \mathrm{CI}, 0.27-1.60 ; P=.36$ ). Within the large LA group only, multivariate analysis found that longer duration of $\mathrm{AF}$ (years) was the only independent predictor for $\mathrm{AF}$ at 1 year (OR, 1.22; 95\% CI, 1.01-1.47; $P=.04)$.

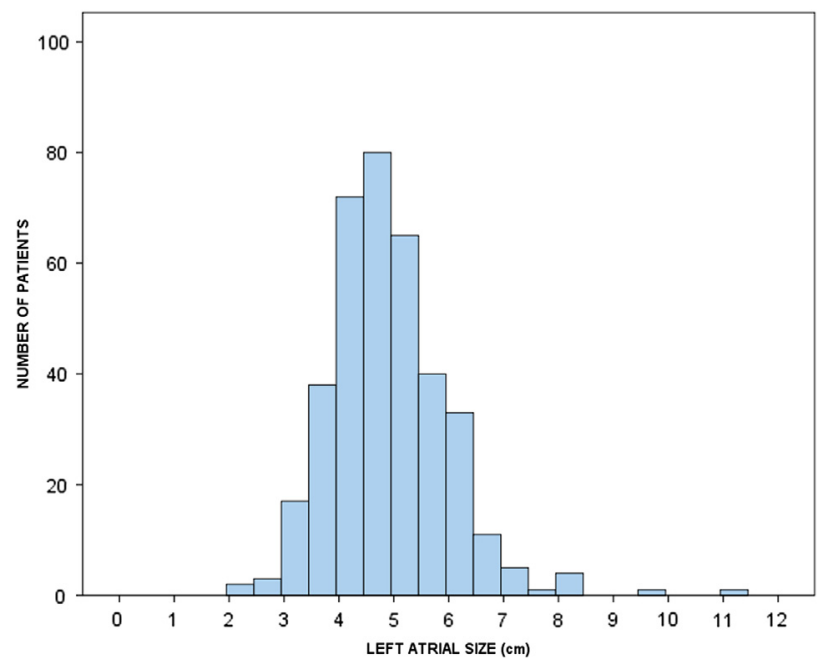

FIGURE 2. Histogram demonstrating left atrial size (centimeters) distribution across the sample. 


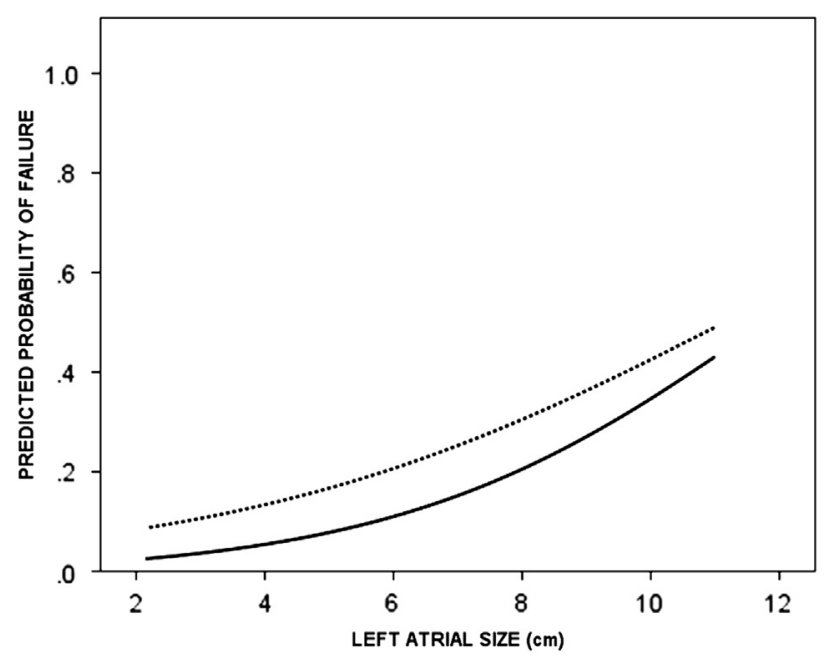

FIGURE 3. Predicted probability of failure (AF) at 12 months (solid line) and failure (AF or normal SR on antiarrhythmic medications) at 12 months (dashed line) across left atrial size as a continuous variable.

\section{DISCUSSION}

An enlarged LA does convey a decreased probability of being in SR at 12 months. However, despite the decreased likelihood of being in SR at 12 months, this group still experienced a high rate of return to SR at 12 months ( $86 \%$ in SR), as well as a relatively high rate of return to SR without antiarrhythmic medications $(77 \%)$ ). By 2 years after the Cox-Maze procedure, the likelihood of being in SR with and without medications was similar for both groups $(>85 \%$ and $>73 \%$, respectively). The postoperative outcomes were comparable for both patient groups with few complications incurred. Even the group of patients with an exceedingly large LA $(\geq 7.5 \mathrm{~cm})$ obtained an acceptable return to SR whether on or off antiarrhythmic drugs. In addition, embolic stroke events were low in both

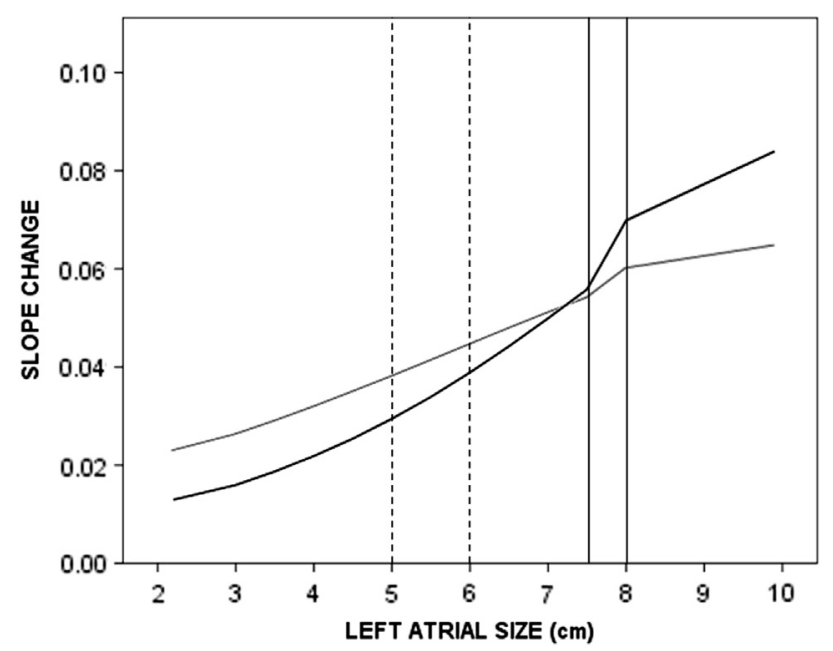

FIGURE 4. Change in slope for the univariate relationship between left atrial size and failure shown in Figure 3.
LA groups despite the majority of patients no longer anticoagulated at 12 months post-surgery.

Patients with a larger LA were found to have significantly more mitral valve disease. This observation has been described in previous studies ${ }^{15,16}$ in which patients with mitral valve disease who had on average an LA greater than $5.5 \mathrm{~cm}$ experienced excellent outcomes after surgical ablation. Researchers have also found that patients who have their AF addressed at the time of surgery have better long-term survival when compared with patients in whom $\mathrm{AF}$ was not addressed at the time of surgery. ${ }^{17}$

In the multivariate analysis for the large LA group, the only predictor for failure to establish SR at 12 months was the duration of AF. This finding is congruent with others who have reported on the failure of the Cox-Maze procedure or surgical ablation because duration of $\mathrm{AF}$ is closely associated with the development of atrial substrate modification. Qian and colleagues, ${ }^{18}$ when investigating the occurrence of AF with mitral valve disease, found that the LA in this group of patients had a significantly greater amount of fibrosis than those who presented with mitral valve disease only.

Schotten and colleagues ${ }^{19}$ have been studying the translational approach to the pathophysiologic mechanisms of AF. They have found that atrial remodeling, which occurs as a result of the electrical, contractile, and structural changes within the atria, starts early even before the first episode of AF occurs. They have recommended an individual early intervention approach based on the numerous pathophysiologic processes that take place when AF occurs. ${ }^{19}$ In a prospective study of 263 patients undergoing operation for mitral valve disease and AF, Gillinov and co-investigators ${ }^{9}$ determined that the duration of $\mathrm{AF}$ was a significant predictor of failure of the Cox-Maze procedure. ${ }^{9}$ They concluded that earlier intervention also was warranted in those with $\mathrm{AF}$ and mitral valve disease to increase the chance of SR restoration and to decrease the chance for AF recurrence.

Because of the different substrate changes associated with mitral valve disease and subsequently the development of AF, atrial tachyarrhythmias can be an issue after the Cox-Maze procedure. Saint and colleagues ${ }^{15}$ studied this phenomenon and found that patients who had mitral valve disease and $\mathrm{AF}$ and underwent mitral valve surgery and a Cox-Maze procedure had more tachyarrhythmias in the first few months after surgery, but by 12 months their results were similar to those of patients who had a Cox-Maze procedure for lone AF. We found that the majority of patients with a large LA had mitral valve disease and a lower return to SR at 1 year off antiarrhythmic drugs, but by 2 years the rate of return to SR was the same as the patients without an enlarged LA, suggesting possible reversal of the atrial remodeling.

Sunderland and colleagues ${ }^{10}$ recently published a best evidence article on what size of the LA impairs the success 
of a surgical ablation procedure for AF. After reviewing 422 articles, with 12 studies that met all the criteria related to the research question, they found that the significant cutoff point for the success of a surgical ablation procedure was $6.0 \mathrm{~cm}$. They recommended caution be exercised when offering a surgical ablation procedure to patients with an LA greater than $6.0 \mathrm{~cm}$. They also found, as various models of AF suggest, that reducing atrial mass or diameter may improve the outcome of surgical ablation by abolishing the potential for reentry circuits and recurrence of AF. Furthermore, because it was uncommon to find surgical patients with a left atrial diameter less than $4 \mathrm{~cm}$, the physiologic basis for atrial reduction surgery is supported in aiding the success of a Cox-Maze procedure. The evidence suggests that patients with an enlarged LA who are at higher risk of failure after standard surgical ablation procedures may gain benefit from concomitant atrial reduction surgery. However, the evidence is not strong enough because all the studies are lacking a comparable group with no left atrial reduction procedure, and there are significant variations in the populations and procedures involved. Therefore, there is a need for prospective randomized studies in this area.

We also found that the rate of failure increased when the LA was greater than $5.5 \mathrm{~cm}$, although this was only significant at 1 year. In addition, an encouraging finding in our study was that the patients with an LA $7.5 \mathrm{~cm}$ or greater had a high rate of return to SR. This finding warrants further study especially because the majority of our patient population underwent the full Cox-Maze procedure, and the report by Sunderland and colleagues ${ }^{10}$ included different surgical ablation procedures, ranging from a limited left-sided only ablation to the full Cox-Maze procedure.

We believe a confounding factor in this study relates to our thinking that $\mathrm{AF}$ is a chronic disease that requires close lifelong follow-up. In line with this philosophy, we developed a clinical protocol to treat patients after a surgical ablation procedure. ${ }^{20}$ Our protocol addresses patients who have returned to SR and the timely discontinuation of their antiarrhythmic and anticoagulation therapy when warranted. The protocol also addresses any recurrence of atrial arrhythmia appropriately through medical therapy or further intervention to obtain SR. Details on our protocol and its impact on the restoration of SR after the Cox-Maze procedure or surgical ablation have been published., 21,22 We believe that using a follow-up protocol is essential in assessing and monitoring for the recurrence of atrial arrhythmias and addressing issues associated with anticoagulation. Our experience shows that the clinical protocol is safe and effective, resulting in improved outcomes. Therefore, it is important to implement and use such protocols in patients who are more prone to have failure after surgical ablation for AF, such as the subgroup of patients with increased left atrial size.

\section{Study Limitations}

This is a single-center study in which more than 700 surgical ablation procedures have been performed by experienced surgeons. These results may not be replicated in centers with a lower patient volume.

\section{CONCLUSIONS}

The large LA group $(>5.5 \mathrm{~cm})$ had an acceptable rate of SR restoration overall and without antiarrhythmic medications. Embolic stroke events were low in both groups despite the majority of patients no longer taking anticoagulation medication. If patients are managed appropriately after surgical ablation, acceptable results can be expected with the added advantage of lower risk of stroke and need for anticoagulation. Our findings suggest that left atrial size should not be a discouragement when evaluating a surgical candidate with AF.

\section{References}

1. Cox JL, Schuessler RB, D’Agostino HJ Jr, Stone CM, Chang BC, Cain ME, et al The surgical treatment of atrial fibrillation. III. Development of a definitive surgical procedure. J Thorac Cardiovasc Surg. 1991;101:569-83.

2. Ad N, Henry L, Hunt S. The concomitant cryosurgical Cox-Maze procedure using Argon based cryoprobes: 12 month results. J Cardiovasc Surg (Torino). 2011;52:593-9.

3. Cox JL, Ad N, Palazzo T, Fitzpatrick S, Suyderhoud JP, DeGroot KW, et al. Current status of the Maze procedure for the treatment of atrial fibrillation. Semin Thorac Cardiovasc Surg. 2000;12:15-9.

4. Melby SJ, Zierer A, Bailey MS, Cox JL, Lawton JS, Munfakh N, et al. A new era in the surgical treatment of atrial fibrillation: the impact of ablation technology and lesion set on procedural efficacy. Ann Surg. 2006;244:583-92

5. Ad N. The multi-purse string Maze procedure: a new surgical technique to perform the full Maze procedure without atriotomies. J Thorac Cardiovasc Surg. 2007; 134:717-22.

6. Weimar T, Schena S, Bailey MS, Maniar HS, Schuessler RB, Cox JL, et al. The Cox-Maze procedure for lone atrial fibrillation: a single-center experience over 2 decades. Circ Arrhythm Electrophysiol. 2012;5:8-14

7. Damiano RJ Jr, Schwartz FH, Bailey MS, Maniar HS, Munfakh NA, Moon MR, et al. The Cox Maze IV procedure: predictors of late recurrence. J Thorac Cardiovasc Surg. 2011;141:113-21

8. Romano MA, Bach DS, Pagani FD, Prager RL, Deeb GM, Bolling SF. Atrial reduction plasty Cox Maze procedure: extended indications for atrial fibrillation surgery. Ann Thorac Surg. 2004;77:1282-7.

9. Gillinov AM, Sirak J, Blackstone EH, McCarthy PM, Rajeswaran J, Pettersson G, et al. The Cox Maze procedure in mitral valve disease: predictors of recurrent atrial fibrillation. J Thorac Cardiovasc Surg. 2005;130:1653-60.

10. Sunderland N, Maruthappu M, Nagendran M. What size of left atrium significantly impairs the success of Maze surgery for atrial fibrillation? Interact Cardiovasc Thorac Surg. 2011;13:332-8.

11. Gaynor SL, Schuessler RB, Bailey MS, Ishii Y, Boineau JP, Gleva MJ, et al. Surgical treatment of atrial fibrillation: predictors of late recurrence. J Thorac Cardiovasc Surg. 2005;129:104-11.

12. Cox JL. The surgical treatment of atrial fibrillation. IV. Surgical technique. J Thorac Cardiovasc Surg. 1991;101:584-92.

13. Calkins H, Kuck KH, Cappato R, Brugada J, Camm AJ, Chen SA, et al. 2012 HRS/EHRA/ECAS expert consensus statement on catheter and surgical ablation of atrial fibrillation: recommendations for patient selection, procedural techniques, patient management and follow-up, definitions, endpoints, and research trial design. J Interv Card Electrophysiol. 2012;33:171-257.

14. Sunderland N, Nagendran M, Maruthappu M. In patients with an enlarged left atrium does left atrial size reduction improve Maze surgery success? Interact Cardiovasc Thorac Surg. 2011;13:635-41.

15. Saint LL, Bailey MS, Prasad S, Guthrie TJ, Bell J, Moon MR, et al. Cox-Maze IV results for patients with lone atrial fibrillation versus concomitant mitral disease. Ann Thorac Surg. 2012;93:789-95 
16. Ad N, Cox JL. Combined mitral valve surgery and the Maze III procedure. Semin Thorac Cardiovasc Surg. 2002;14:206-9.

17. Gillinov AM, Saltman AE. Ablation of atrial fibrillation with concomitant cardiac surgery. Semin Thorac Cardiovasc Surg. 2007;19:25-32.

18. Qian Y, Meng J, Tang H, Yang G, Deng Y, Wei D, et al. Different structural remodelling in atrial fibrillation with different types of mitral valvular diseases. Europace. 2010;12:371-7.

19. Schotten U, Verheule S, Kirchhof P, Goette A. Pathophysiological mechanisms of atrial fibrillation: a translational appraisal. Physiol Rev. 2011;91:265-325.
20. Ad N, Henry L, Hunt S, Stone L. The implementation of a comprehensive clinica protocol improves long-term success after surgical treatment of atrial fibrillation. J Thorac Cardiovasc Surg. 2010;139:1146-52

21. Ad N, Henry L, Schlauch K, Holmes SD, Hunt S. The CHADS score role in managing anticoagulation after surgical ablation for atrial fibrillation. Ann Thorac Surg. 2010;90:1257-62.

22. Henry LL, Ad N, Martin L, Hunt S, Crippen P. A quality improvement project to optimize patient outcomes following the Maze procedure. J Nurs Care Qual. 2009;24:160-5. 